\title{
Approaches to Broaden Messaging about the Harms Tobacco Use
}

\author{
Gail Cherry-Peppers, Andrea D Jackson, Cheryl Fryer, Xinbin Gu, Jezelle Sonnier, Dorienne \\ Taylor-Bishop, Paula Nesbitt, Lisa Slade, Meirong Liu, Gretchen Peppers and Gillian Robinson- \\ Warner* \\ Department of Dentistry, Howard University School of Social Work, USA
}

*Corresponding author: Gillian Robinson-Warner, Department of Restorative Dentistry, Howard University School of Social Work, USA.

To Cite This Article: Gail Cherry-Peppers, Andrea D Jackson, Cheryl Fryer, Xinbin Gu, Gillian Robinson-Warner, et al., Approaches to Broaden Messaging about the Harms Tobacco Use. Am J Biomed Sci \& Res. 2021 - 14(2). AJBSR.MS.ID.001962. DOI: 10.34297/AJBSR.2021.14.001962.

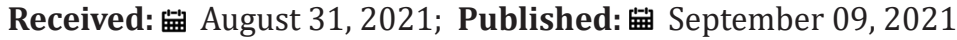

\begin{abstract}
Dentist and other health professionals can have great influence to prevent and decrease the use tobacco products, through patient visits, direct messaging and social media. A brief part of the dental and medical visit can be used for providing current, state-of-the-art research and information on the harms of e-cigarette and cigarette use and the use of smokeless tobacco products. Many studies have shown that various tobacco products contain toxicants, constituents and harmful chemicals. Variations of different combinations of tobacco-related toxicants pass through the oral mucosal tissues, enter body fluids and systems causing severe health effects. During the assessment of findings and sharing of visual materials segment of the visit, the health professional can present the information so that the patient can better understand the physiological changes that may occur systemically, and the morbidity and mortality that may be associated with long-term tobacco use. Evidence-based research on the adverse health effects of tobacco use can be shared solely with the patient, and broadly through social media.
\end{abstract}

Keywords: Tobacco Product Use; Carcinogens; Tobacco-Related Diseases; Patient Education; Oral Health

\section{Introduction}

This review will raise awareness provide information that can be shared with patients about the chronic exposure to carcinogens. Chemical elements in tobacco pass through the oral mucosa which can result in an increased risk of oral cancer, other diseases, addiction, and exposure to toxicants. These dysplastic changes are often a result of frequency and duration of long-term product tobacco use [1]. The mouth is one of the few rare body areas in which health professionals can sometimes detect early lesions and areas of concern upon visual examinations. Health care providers can identify early signs of a disease state and assess levels of progression during each oral examination visit. Thirty percent to eighty percent of the malignancies of the oral cavity arise from premalignant lesions, such as leukoplakia, erythroplakia and oral submucous fibrosis, which may form due to long-term use of cigarettes, new and emerging smokeless tobacco and e-cigarettes
$[2,3]$. Pictures and graphics are also good visual tools to share with patients, to update them on tobacco-related disease, oral cancer, and other resulting oral health conditions due to tobacco use. Dentists play a key role in messaging, sharing facts verbally, through telehealth mechanisms and social media. Providing key facts about long-term tobacco use and harm provide the patient a better understanding of their slow path to poor health.

\section{Harmful Effects of Tobacco Use}

In 2020, an estimated 54,000 adults in the United States were diagnosed with oral cavity and oropharyngeal cancer, and an estimated 10,700 people will die of these cancers. The 5-year survival rate for oral and pharyngeal cancer is approximately $50 \%-57 \%$ [4]. The use of smokeless tobacco is associated with oral cancer and is commonly diagnosed in the later stages of the disease $[1,5]$. The use of conventional cigarettes has numerous oral and systemic manifestations, which have been studied for more 
than 50 years. N'-nitrosonornicotine (NNN), an important tobaccospecific nitrosamine (TSNA), is found in conventional cigarettes and smokeless tobacco products [6,7]. E-cigarettes are associated with some of the same adverse health risks related to cigarettes, such as poor periodontal health, lung conditions, and risk for oral cancer.

The carcinogen NNN has been detected in the saliva of e-cigarette users. NNN may cause oral and esophageal cancer [68]. Poly-tobacco use to include E-cigarettes, conventional cigarette uses and smokeless tobacco use is growing rapidly in popularity. Early identification of oral mucosal cellular tissue alterations and other oral disease conditions will result in the reduction of deleterious health effects. The harmful constituents found in cigarettes, smokeless tobacco, and e-cigarettes contain chemicals that are dangerous, and many of these toxicants are triggers for the progression of carcinogenesis [1].

\section{Trends and Patterns of Tobacco Use}

Conventional cigarette use has greatly declined over the past 50 years (Figure 1). Consumption of tobacco products have increased globally (ash.org/programs/statistics). Approximately 34 million people currently smoke cigarettes (14\%). Long-term smoking causes cancer, heart disease, lung disease, emphysema, chronic obstructive pulmonary disease (COPD), and increases the severity of diabetes and other diseases. Oral health effects include oral cancer, periodontal disease, higher untreated tooth decay, root caries, slow healing, and greater loss of teeth $[9,10]$. There are over 7,000 toxic chemicals in tobacco, which makes it a large driver of cancer, particularly oral cancer [11] https://ash.org/ programs/statistics. In a study by Jeong et al. [12] it was noted that E-cigarette users and those who used cigarettes were more likely to have periodontal disease, regardless of whether they lived in in an urban or rural area, or regardless of education level [12]. E-cigarette use has grown exponentially in the US since 2007. Easily concealed devices make it difficult for parents to monitor e-cigarette use in many adolescents. Health effects such as altered brain development in teens are one of a few long-term health effects of concern [13]. In a study by Bustamante et al in was noted that 16 out of 20 e-cigarette users had measurable levels of NNN in their saliva. The levels were generally lower than in smokers, but some e-cigarette users had NNN in their saliva. Thus, harmful constituents such as NNN found in saliva can result in diminishing health outcomes [8]. Growing popularity for dual and poly-tobacco product use, particularly in teens and young adults is a public health burden. Chemicals from the use of a single product increase the risk of oral and other cancers. Dual and multi-product may further heighten the risk of developing various tobacco- related diseases and conditions. Broadened awareness about the dangers of multi-tobacco product use through increased patient education efforts, may reduce long-term harm and disease. Polytobacco use substantially increases exposure to toxicants [14]. Many studies have shown that, independent of the pattern of tobacco use, and nicotine yields, levels of TSNA's in many tobacco products play a significant role in carcinogen exposure levels $[7,15]$. Studies have also shown that cessation of tobacco smoking can lower the risk of head and neck cancer. Moreover, cigarette smokers who are dual/polyusers may smoke as many or more cigarettes per day as exclusive cigarette smokers, thereby increasing their risk of poor health outcomes by using additional tobacco products [14].

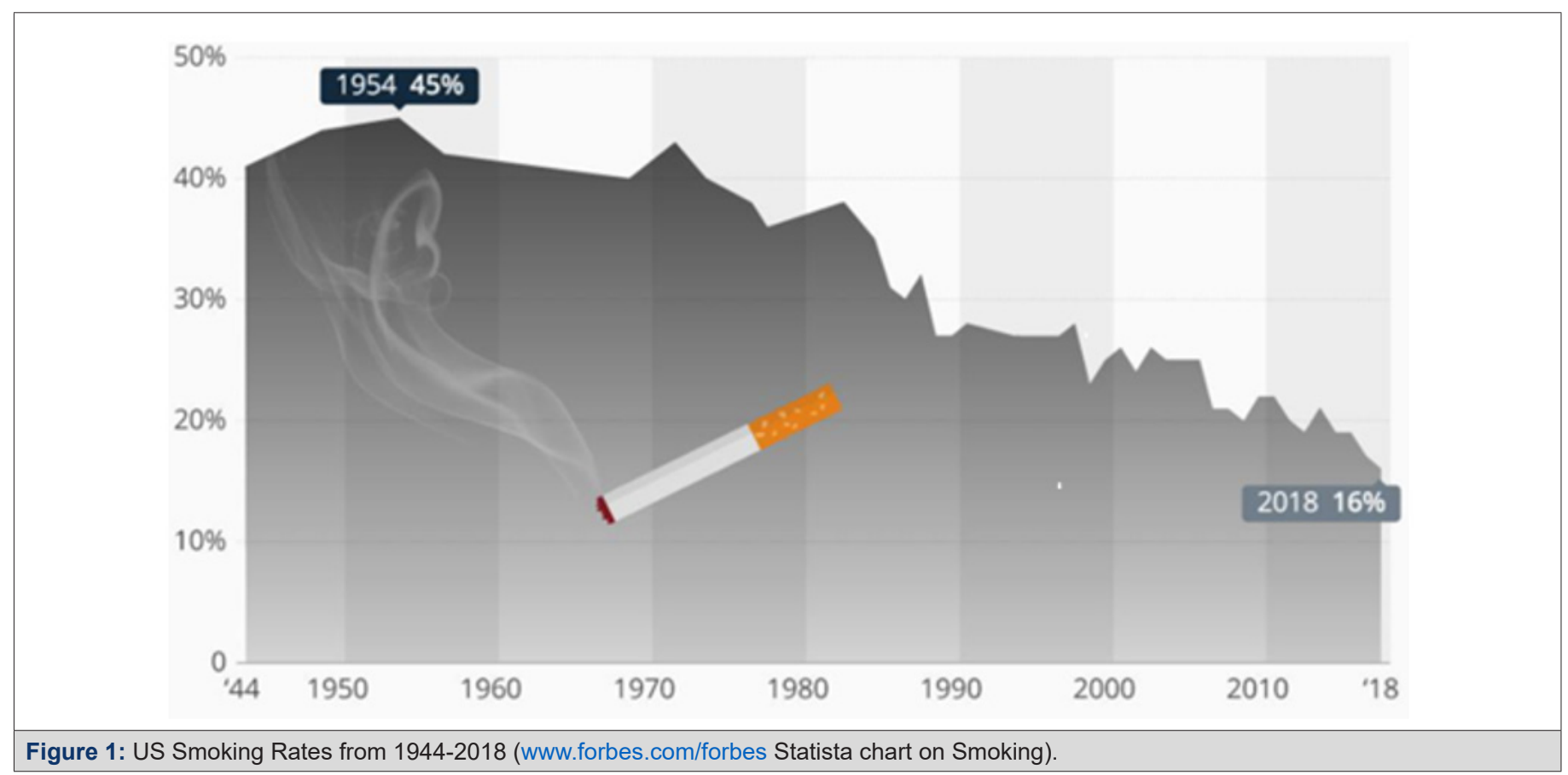


Users of smokeless tobacco and users of cigarettes have comparable levels of nicotine in the blood. Nicotine which is absorbed through the oral cavity tissues directly continues into the blood, where it goes to the brain. Even after the tobacco is removed from the mouth, nicotine continues to be absorbed into the bloodstream [16]. Orally absorbed nicotine stays in the blood longer for users of smokeless tobacco, than for cigarette smokers and is easily absorbed through the oral mucosal lining. Approximately twice as much as nicotine is absorbed per dose of smokeless tobacco than cigarettes ( $4 \mathrm{mg}$ vs. $2 \mathrm{mg}$ ). It is estimated that one pouch of smokeless tobacco currently on the market contains on average about twenty times lower amounts of analyzed agents than an average portion of traditional products. Smokeless tobacco products deliver substantial doses of nicotine along with powerful cancer-causing chemicals. Nicotine from e-cigarette use varies per user. Long-term use of e-cigarette toxicants can yield damaging effects $[17,18]$. Absorption of nicotine across biological membranes due to cigarette smoke, smokeless tobacco or E-cigarettes is highly $\mathrm{pH}$ dependent. Free nicotine (unionized and $\mathrm{PH}$ above 6.5) is readily absorbed into biological tissues, and well absorbed through the mouth and buccal membranes. The significance of the transfer of tobacco-related constituents and toxicants across oral mucosal membranes increases the potential for systemic diseases, oral diseases and many forms of cancer [18-20]. Constituents cross oral mucosal membranes, enter the bloodstream and subsequently the brain where is exerts the pharmacological effects that may lead to addiction [20]. The results of two studies by Hatsukami. et al. confirm that the frequency, duration, the amount of product used, residence, time and flux within the mouth cavity affect nicotine exposure [21] from cigarettes, smokeless tobacco and e-cigarettes.

\section{How Dentists can Impact and Better Communicate to Patients about Harms of Tobacco Use}

The oral cavity can serve as a useful indicator of health and disease. Early detection of oral and systemic conditions can lead to decreased mortality. Dental health professionals and their medical interprofessional team can greatly influence patients by increasing communication about the science behind tobacco use. Dental professionals must disclose findings such as oral lesions, areas of irritation, increased pocket depth, bone loss, tooth loss, increased caries, gingival inflammation, stains, heavy tartar build-up, and red or irritated tissues along the oral-pharyngeal areas to the patient [10]. Tobacco users should be closely followed, provided new and emerging research on the association of oral findings and tobacco use, and consulted about the need to discontinue smoking cigarettes, using smokeless tobacco products and/or e-cigarettes. If conditions are extensive, a medical consultation should be requested.

\section{Conclusion}

As the population is living longer in an environment of advanced technology, many patients who are long-term tobacco users have complications that can be treated early, and the patient's dental and medical team can ultimately improve overall clinical care. Detailed medical consultations will afford the dentist an opportunity to adjust treatment planning and execution and avoid serious complications, as they are on the front lines to battle oral diseases. Discussions with patients about the harms of tobacco use may result in decreased or total discontinuance of various tobacco products. The patient can be provided information in which they can use to make informed decisions as to whether to continue high risk behaviors. Use of the oral examination during the dental/ medical visit and clear messaging about the numerous adverse health conditions due to single, dual and poly tobacco product use are critical in the fight to eliminate all tobacco use. Social media is a tool that can be increasingly used to share information on the harms of polytobacco use and reach the population under 50 years of age. Addressing the difficult challenge to change behaviors of individuals who use tobacco is a step forward in the elimination of tobaccorelated diseases and death. The challenge for health professionals is in integrating and taking the time to provide scientific updates which may alert the patient to make better lifestyle decisions. Social media posts can broaden the evidence knowledge base, expand the reach to numerous communities and invoke consideration to cease all forms of tobacco use.

\section{References}

1. Neville BW, Day TA (2002) Oral Cancer and Precancerous Lesions. CA Cancer J Clin 52(4): 195-215.

2. Shulman JD, Beach MM, Rivera-Hidalgo F (2004) The prevalence of oral mucosal lesions in US adults: data from the Third National Health and Nutrition Examination Survey, 1988-1994. J Am Dent Assoc 135(9): 1279-1286.

3. Taybos G (2003) Oral changes associated with tobacco use. Am J Med Science 326(4): 179-182.

4. Oral Cancer Facts.

5. Stepanov I, Hatsukami D (2016) Call to Establish Constituent Standards for Smokeless Tobacco Products. Tobacco Regulatory Sci 9-30(22).

6. Health Harms from Smokeless Tobacco Use.

7. Stepanov I, Knezevich A, Zhang L (2012) Carcinogenic Tobacco-Specific N-Nitrosamines in U.S. Cigarettes-Three Decades of Remarkable Neglect by the Tobacco Industry. Tob Control 21(1): 44-48.

8. Bustamante G, Ma B, Yakovlev G, Yershova K, Le C (2018) Presence of the Carcinogen N'-Nitrosonornicotine in Saliva of E-cigarette Users. Chem. Res. Toxicol 31: 731-738.

9. Centers for Disease control and prevention.

10. Couch E, Chaffee BW, Gansky SA, Walsh MM (2016) The Changing Tobacco Landscape-What Dental Professionals Need to Know. Journal of the Amer Dental Assoc 147(7): 561-569. 
11. Global Action for everyone's Health.

12. Jeong W, Choi DW, Kim YK, Lee HJ, Lee SA, et al. (2020) Associations of Electronic and Conventional Cigarette Use with Periodontal Disease in South Korean Adults. Jour Periodontology 91(1): 55-64.

13. Tomar SL, Fox CH, Connolly GN (2015) Electronic Cigarettes: The Tobacco Industry's Latest Threat to Oral Health. Jour of the Amer Dental Assoc 146(9): 651-653.

14. Hirschtick JL, Mattingly DT, Cho B, Arciniega LZ, David L Hirschtick, et al. (2021) Exclusive, Dual and Polytobacco Use Among US Adults by Sociodemographic Factors: Results From 3 Nationally Representative Surveys. American Journal of Health Promotion 35(3): 377-387.

15. Hatsukami DK, Stepanov I, Severson H (2015) Evidence Supporting Product Standards for Carcinogens in Smokeless Tobacco. Cancer Prevention Research 8(1): 20-26.
16. Newell J (2001) Tobacco Use and Oral Cancer: A Global Perspectivve. J Dental Educ 65(4): 328-339.

17. Covid-19 Resources. National Cancer Institute.

18. Tomar SL, Henningfiled JE (1997) Review of the Evidence That $\mathrm{P}^{\mathrm{H}}$ Is a Determinant of Nicotine Dosage from Oral Use of Smokeless Tobacco. Tob Control 6(3): 219-225.

19. Greer RO (2011) Oral Manifestations of Smokeless Tobacco Use. Otolaryngology Clin of North America 44(1): 31-56.

20. Wiener RC (2013) Association of Smokeless Tobacco Use and Smoking in Adolescent in the U.S. Youth Risk Behavior Survey. Jour American Dent Assoc 144(8): 930-938.

21. Hatsukami DK, Keenan RM, Anton, DJ (1988) Topographical Features of Smokeless Tobacco Use. Psychopharmacology 96(3): 428-442. 